samples are collected to evaluate $\mathrm{T}$ cell responses using flow cytometry, ELISA, ELISPOT.

Results As of July 23, 2020, 9 patients were enrolled. Median duration of ongoing anti-PD therapy was 37 weeks (range 20101). The combination was well-tolerated with no DLTs and mostly Grade 1-2 unrelated adverse events. Two Grade 3 events were reported: hypertension (not related) and dehydration (related), the later reported as serious adverse event. Of seven patients eligible for efficacy analysis, one patient with PD-L1 negative disease had a partial response with a reduction of $29 \%$ at week 6 with deepening of the response to $43 \%$ at week 12 and one patient with progressive disease at study entry had stabilization of disease at week 6 and 12 . Another two patients had stable disease for $30+$ weeks and three patients had PD. Additional efficacy and immunological analyses are ongoing.

Conclusions Early data show that the combination of SNS-301 and pembrolizumab has manageable toxicity and capacity to achieve long-term disease stability and objective tumor responses.

Trial Registration NCT04034225

Ethics Approval This study has been approved by WIRB (20190628) as well as several institutional IRBs.

http://dx.doi.org/10.1136/itc-2020-SITC2020.0349

\section{PEMBROLIZUMAB PLUS LENVATINIB VS CHEMOTHERAPY AND LENVATINIB MONOTHERAPY FOR RECURRENT/METASTATIC HEAD AND NECK SQUAMOUS CELL CARCINOMA THAT PROGRESSED ON PLATINUM THERAPY AND IMMUNOTHERAPY: LEAP-009}

${ }^{1}$ Kevin Harrington* ${ }^{2}$ Ezra Cohen ${ }^{3}$ ilian Siu, ${ }^{4}$ Danny Rischin ${ }^{5}$ isa Licitra ${ }^{6}$ Jan Vemo ${ }^{7}$ Quynh-Thu Le, ${ }^{8}$ Makoto Tahara, ${ }^{9}$ Jean-Pascal Machiels, ${ }^{10}$ Natalyn Hawk, ${ }^{11}$ Joy Ge, ${ }^{11}$ Behzad Bidadi, ${ }^{11}$ Ramona Swaby, ${ }^{12}$ Barbara Burtness. ${ }^{1}$ The Institute of Cancer Research, London, UK; ${ }^{2}$ University of California San Diego and Moores Cancer Center, La Jolla, CA, USA; ${ }^{3}$ University Health Network, Toronto, ON, Canada; ${ }^{4}$ Peter MacCallum Cancer Centre, East Melbourne, Australia; ${ }^{5}$ Fondazione IRCCS Istituto Nazionale dei Tumori and University of Milan, Milan, Italy; ${ }^{6}$ Antwerp University Hospital, Edegem, Belgium; ${ }^{7}$ Stanford University, Stanford, CA, USA; ${ }^{8}$ National Cancer Center Hospital, Kashiwa, Japan; ${ }^{9}$ Université catholique de Louvain, Brussels, Belgium; ${ }^{10}$ Eisai Inc., Woodcliff Lake, NJ, USA; ${ }^{11}$ Merck and Co., InC., Kenilworth, NJ, USA; ${ }^{22}$ Yale Cancer Center, New Haven, CT, USA

Background Pembrolizumab alone and in combination with platinum-based chemotherapy have become standard first-line treatment options for recurrent/metastatic head and neck squamous cell carcinoma (R/M HNSCC), and there is a growing unmet need for safe and efficacious treatment options for $\mathrm{R} /$ M HNSCC that has progressed on or after platinum-based chemotherapy and immunotherapy. Data from Study 111/KEYNOTE-146 showed promising antitumor activity and acceptable safety for the PD-1 inhibitor pembrolizumab given in combination with the multikinase inhibitor lenvatinib in patients with metastatic HNSCC. ${ }^{1}$ LEAP-009 (NCT04428151), a global, randomized, open-label, phase 2 trial, will assess the efficacy and safety of pembrolizumab in combination with lenvatinib versus SOC chemotherapy, as well as the efficacy and safety of lenvatinib monotherapy, in patients with $\mathrm{R} / \mathrm{M}$ HNSCC that has progressed after platinum-based chemotherapy and a PD-(L)1 inhibitor.

Methods Eligible patients are adults with histologically confirmed, locally incurable R/M HNSCC of the oral cavity, oropharynx, hypopharynx, or larynx, disease progression at any time during or after platinum-containing chemotherapy (with or without cetuximab), disease progression within 12 weeks from the last dose of treatment with $\geq 2$ doses of a PD-(L)1 inhibitor, measurable disease based on RECIST v1.1 as confirmed by BICR, ECOG performance status of 0 or 1 , and no major blood vessel invasion/infiltration. Patients will be randomized 3:3:2 to pembrolizumab (200 mg IV Q3W for up to 35 cycles) plus lenvatinib ( $20 \mathrm{mg}$ orally once daily), investigator's choice of SOC chemotherapy (docetaxel, paclitaxel, cetuximab, or capecitabine), or lenvatinib monotherapy (24 mg orally once daily). Randomization will be stratified by PDL1 tumor proportion score $(<50 \%$ versus $\geq 50 \%)$ and ECOG performance status (0 versus 1$)$. Treatment will continue until centrally verified disease progression, unacceptable toxicity, or decision to withdraw. Patients in the chemotherapy and lenvatinib monotherapy arms may be eligible to receive pembrolizumab plus lenvatinib upon disease progression. The primary endpoint is ORR according to modified RECIST v1.1 as assessed by BICR. Secondary endpoints include PFS, OS, DOR, and safety. Interim futility analysis will be conducted for the lenvatinib monotherapy arm. Tumor imaging by CT or MRI will be performed 6 weeks after randomization, every 6 weeks through year 1 , and every 9 weeks thereafter. Safety will be monitored throughout the study and for 30 days after treatment end (90 days for serious AEs if no new anticancer treatment is initiated, and at any time if the $\mathrm{AE}$ is considered treatment-related). Recruitment is ongoing; Planned enrollment is $\sim 400$ patients.

Results N/A

Conclusions N/A

Trial Registration ClinicalTrials. gov Identifier, NCT04428151 Ethics Approval The study and protocol were approved by the Institutional Review Board or ethics committee at each site. Consent All patients provided written informed consent to participate in the clinical trial.

\section{REFERENCE}

1. Matthew H Taylor, Chung-Han Lee, Vicky Makker, et al. Phase IB/II trial of lenvatinib plus pembrolizumab in patients with advanced renal cell carcinoma, endometrial cancer, and other selected advanced solid tumors. J Clin Oncol 2020;38 (11):1154-1163.

http://dx.doi.org/10.1136/jitc-2020-SITC2020.0351

\section{UPDATED CLINICAL DATA FROM THE SQUAMOUS CELL CARCINOMA OF THE HEAD AND NECK (SCCHN) EXPANSION COHORT OF AN ONGOING PH1/1B STUDY OF EGANELISIB (FORMERLY IPI-549) IN COMBINATION WITH NIVOLUMAB}

${ }^{1}$ Ezra Cohen*, ${ }^{2}$ Michael Postow, ${ }^{3}$ Ryan Sullivan, ${ }^{4}$ David Hong, ${ }^{5}$ Heather Yeckes-Rodin ${ }^{6}$ Jerry McCarter, ${ }^{6}$ Nora Zizlsperger, ${ }^{7}$ Jeffery Kutok, ${ }^{6}$ Brenda O'Connell, ${ }^{6}$ Kara Page, ${ }^{6}$ Jennifer Roberts, ${ }^{6}$ Halle Zhang, ${ }^{8}$ Bartosz Chmielowski. 'University of California San Diego, La Jolla, CA, USA; ${ }^{2}$ Memorial Sloan Kettering Cancer Center, New York, NY, USA; ${ }^{3}$ Massachusetts General Hospital, Boston, MA, USA; ${ }^{4}$ MD Anderson Cancer Center, Houston, TX, USA; ${ }^{5}$ Heme-Onc Associates of Treasure Coast, Port St. Lucie, FL, USA; ${ }^{6}$ Infinity Pharmaceuticals, Cambridge, MA, USA; ${ }^{7}$ Epizyme, Cambridge, MA, USA; ${ }^{8}$ University of California Los Angeles, Los Angeles, CA, USA

Background Eganelisib is a first-in-class, oral, selective PI3K- $\gamma$ inhibitor. Preclinically, eganelisib reprograms macrophages/myeloid derived suppressor cells (MDSCs) from an immune-suppressive to an immune-activating phenotype and enhances efficacy of checkpoint inhibitors. Efficacy of eganelisib + nivolumab in patients with SCCHN resistant to immediate prior anti-PD(L)1 therapy is presented. 